\title{
Long-term response of gemcitabine plus docetaxel chemotherapy regimen for extraskeletal osteosarcoma: A case report
}

\author{
SABINO STRIPPOLI ${ }^{1}$, MICHELE TRAVERSA $^{2}$, ANTONIO CRAMAROSSA $^{2}$, \\ ONDINA POPESCU ${ }^{3}$, VITO LORUSSO ${ }^{1}$ and MICHELE GUIDA ${ }^{1}$ \\ Departments of ${ }^{1}$ Medical Oncology and ${ }^{2}$ Radiology; ${ }^{3}$ Histopathology Unit, \\ National Cancer Research Centre 'Giovanni Paolo II', Bari 70124, Italy
}

Received July 10, 2014; Accepted February 5, 2015

DOI: $10.3892 / \mathrm{ol} .2015 .3111$

\begin{abstract}
Extraskeletal osteosarcomas (EOSs) are rare variants of primary osteosarcoma of the bone, and are defined as sarcomas located in the soft tissues and characterized by osteoid production. EOS exhibits distinctive demographic, imaging and prognostic features compared with osteosarcoma of bone origin. The available data are contradictory with regard to the use of chemotherapy regimens in the management of EOS. The present study describes a case of EOS that progressed following two lines of therapy oriented to soft-tissue and bone sarcoma histology, respectively. As a gemcitabine-docetaxel combination schedule has demonstrated synergistic activity against bone and soft-tissue sarcoma histologies, this chemotherapy regimen was selected as salvage therapy. The treatment was well-tolerated and induced a long lasting partial response for $\sim 14$ months. To the best of our knowledge, this is the first report involving the clinical use of this combination regimen for the treatment of EOS. Furthermore, as demonstrated in this report, EOS may maintain relative chemosensitivity, indicating the potential to control advanced disease in the long term and to plan subsequent chemotherapy regimens.
\end{abstract}

\section{Introduction}

Extraskeletal osteosarcomas (EOSs) are rare, high-grade malignant mesenchymal tumors of the soft tissue. The tumors are characterized by an osteoid and/or cartilaginous matrix,

Correspondence to: Dr Michele Guida, Department of Medical Oncology, National Cancer Research Centre 'Giovanni Paolo II', 65 Via Orazio Flacco, Bari 70124, Italy

E-mail: micguida@libero.it

Abbreviations: EOS, extraskeletal osteosarcoma; MRI, magnetic resonance imaging; $\mathrm{CT}$, computed tomography; $\mathrm{PR}$, partial response

Key words: extraskeletal osteosarcoma, gemcitabine, docetaxel, salvage therapy which is produced by neoplastic cells with an aberrant osteoblastic phenotype. The distinguishing feature of EOS, which may exhibit similar histological features to primary osteogenic sarcoma of the bone, is the localization in the soft tissues without direct attachment to bone or periosteum. EOSs account for $<5 \%$ of all osteosarcomas and $1 \%$ of all soft-tissue tumors (1-5). Typically EOSs arise as large, deep and painless lesions in the lower extremities, most commonly in the thigh. Unlike skeletal osteosarcoma, which predominantly affects patients in the second and third decades of life, EOSs are frequently observed in patients aged $>50$ years at the time of diagnosis (1-7).

Due to the rarity of EOSs and the features that distinguish them from conventional bone sarcomas, limited reliable data are available with regard to the incidence, clinical behavior and therapeutic management of these tumors. Furthermore, in the era of histology-driven therapy for the treatment of sarcoma subtypes (8), no specific guidelines have been established for the treatment of EOS. In retrospective case studies, contradictory data have been reported regarding the use of chemotherapy regimens indicated for bone $(6,7)$ or soft-tissue sarcoma $(9,10)$.

The present study reports a combination treatment of gemcitabine and docetaxel, utilized as a salvage therapy in a typical case of EOS previously treated with chemotherapy compounds designed either for soft-tissue or bone sarcomas. To the best of our knowledge, no effective salvage therapies have previously been established for patients with recurrent or refractory EOS, and this study is the first to report the use of this combination regimen resulting in synergistic antitumor activity in this disease. Written informed consent was obtained from the patient's family.

\section{Case report}

A 50-year-old male farmer presented to the Oncology Department of the National Cancer Research Centre (Bari, Italy) in July 2011 with a progressively enlarging mass on the medial side of the left proximal thigh. The past medical and family history revealed no illnesses of note. The patient smoked heavily, but did not consume alcohol or use illicit drugs. Although no clear history of trauma was reported, the painless enlarging mass on the thigh was initially misdiagnosed 
A
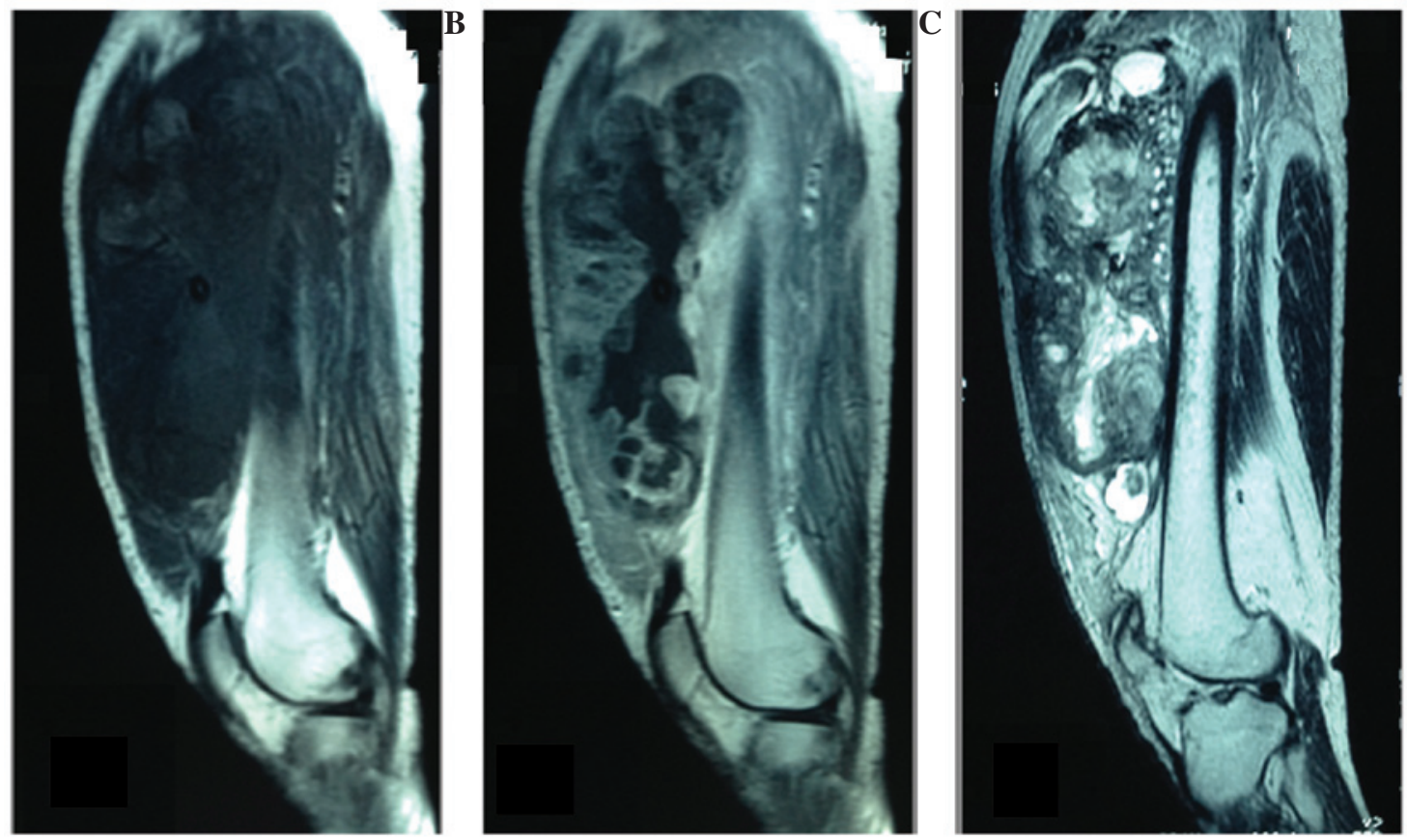

Figure 1. (A) Sagittal T1-weighted MRI showing a large, irregularly lobulated mass, slightly hypointense to skeletal muscle. (B) Sagittal contrast-enhanced T1-weighted MRI revealing heterogeneous contrast enhancement of the tumor with massive central necrosis. (C) Sagittal T2-weighted MRI demonstrating multiple intratumoral cystic areas. MRI, magnetic resonance imaging.

as a hematoma upon physical examination. However, due to the rapid increase in the size and hardness of the palpable mass in the previous month, the patient underwent magnetic resonance imaging (MRI) in August 2011, revealing the presence of a large, well-defined, irregularly lobulated mass in the left quadriceps femoris muscle. The mass was marginally hypointense to skeletal muscle on T1-weighted images and markedly heterogeneously hyperintense on T2-weighted sequences, with patchy intratumoral cystic areas of variable size. The mass had reached a size of $18.5 \times 18.0 \mathrm{~cm}$, and contrast-enhanced MRI and computed tomography (CT) scans revealed irregular contrast enhancement of the tumor with massive central necrosis (Fig. 1).

Results from routine laboratory analyses were within normal limits. Upon physical examination, a hard, bulky mass, which was slightly mobile and not adherent to the skin, was palpable in the left thigh.

An incisional biopsy of the thigh lesion was subsequently performed. Histopathological analysis of the specimen indicated a highly malignant neoplasm with large areas of necrosis, composed of pleomorphic spindle and ovoid cells with a high mitotic rate, which were closely intermingled in a lace-like pattern; intercellular eosinophilic, dense, homogeneous and curvilinear material with focal mineralization was also observed. The neoplastic cells exhibited diffuse, moderate membranous and cytoplasmic positivity for cluster of differentiation 99, diffuse, moderate positivity for smooth muscle actin and focal positivity for S-100. Taken together, these features indicated a diagnosis of EOS (Fig. 2).

As no metastasis was observed on a whole-body CT scan, and in order to attempt subsequent limb-sparing surgery, the patient commenced pre-operative chemotherapy with a regimen consisting of $60 \mathrm{mg} / \mathrm{m}^{2}$ epirubicin on days $1-2$, and
$1,800 \mathrm{mg} / \mathrm{m}^{2}$ ifosfamide on days $1-5$, with mesna (20\% of ifosfamide dose, three times) every 21 days, commencing in September 2011. However, the therapy was discontinued after two cycles due to the onset of local progression, characterized by tumor-associated ulceration and intense swelling of the thigh, and due to the emergence of lung metastases revealed by a further CT scan (Fig. 3A).

The patient experienced progressive anemia caused by bleeding from a lesion of the thigh; a surgical above-knee amputation was therefore conducted to remove the primary EOS. Subsequently, in December 2011, second-line chemotherapy was commenced with $25 \mathrm{mg} / \mathrm{m}^{2}$ doxorubicin on days $1-3$ and $100 \mathrm{mg} / \mathrm{m}^{2}$ cisplatin on day 1 , every 21 days, a regimen typically utilized for the treatment of bone sarcoma (11). Prophylactic polyethylene glycol granulocyte-colony stimulating factor was also administered the day after this chemotherapy. Following three cycles of treatment, a CT scan revealed a partial response (PR) according to the Response Evaluation Criteria In Solid Tumors (version 1.1) (12), with a reduction in the number and size of pulmonary nodules (Fig. 3B). The patient therefore continued this treatment for a further two cycles until May 2012 when, due to a protracted grade 3 pancytopenia, chemotherapy was discontinued. A subsequent CT scan performed in July 2011 showed further PR of the lung lesions and the patient was referred for close follow-up.

In September 2012, a new CT scan indicated disease progression in the lung (Fig. 3C) and the appearance of two nodular brain lesions that were $1 \mathrm{~cm}$ in diameter. Despite an absence of symptoms caused by the brain metastases, the patient underwent whole-brain irradiation. At this time, the patient's Eastern Cooperative Oncology Group status was 0 and in the absence of an established salvage therapy, 

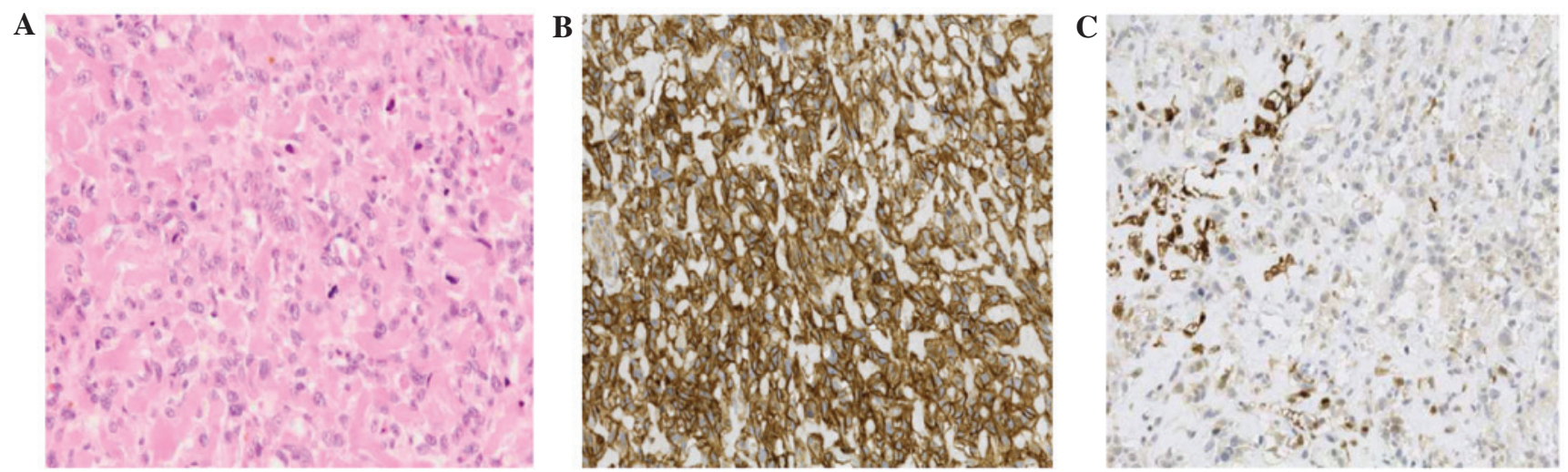

Figure 2. Histopathological appearance of extraskeletal osteosarcoma. (A) Pleomorphic spindle-ovoid cells with focal mineralization, which show (B) diffuse positivity for cluster of differentiation 99 and (C) focal positivity for S-100.
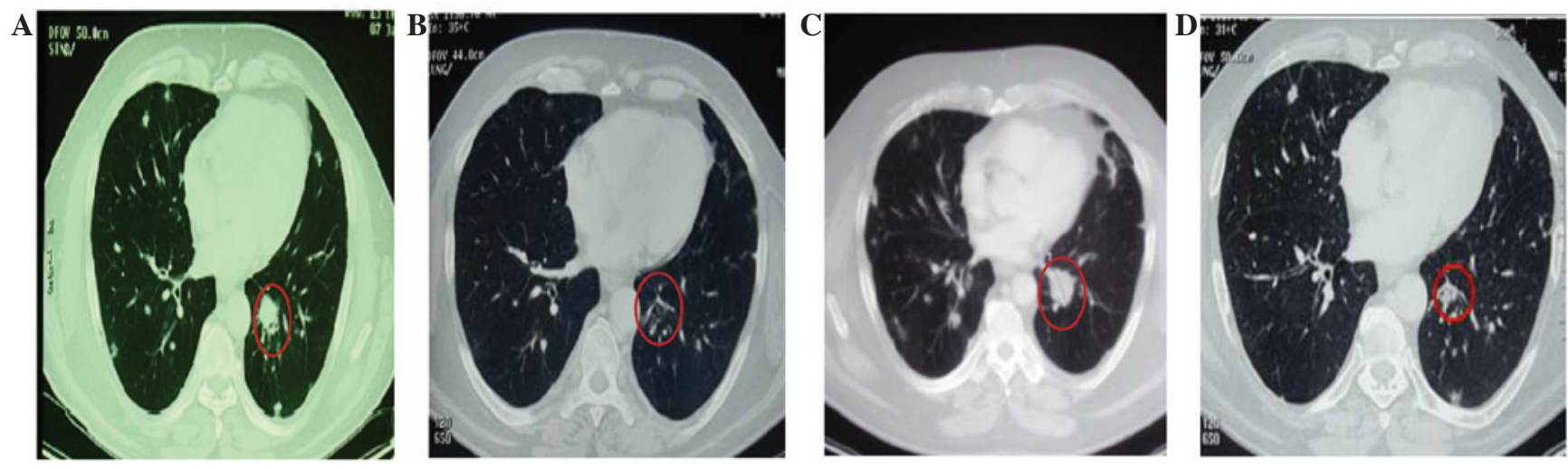

Figure 3. Computed tomography scans revealing lung metastases as the target lesion (red circle). (A) Baseline (prior to the commencement of doxorubicin and cisplatin chemotherapy). (B) Following three cycles of this treatment, when a partial response (PR) was highlighted with an extensive colliquative necrosis phenomenon. (C) At the time of disease progression, prior to the commencement of salvage chemotherapy with gemcitabine and docetaxel. (D) Following 16 cycles of this regimen, when a new PR was achieved.

chemotherapy with $800 \mathrm{mg} / \mathrm{m}^{2}$ gemcitabine and $50 \mathrm{mg} / \mathrm{m}^{2}$ docetaxel was recommenced, administered on day 1 of every two week period. This regimen was selected based on data from a number of studies on the use of this combination regimen in soft-tissue $(11,13-16)$ and bone $(17,14,18-22)$ sarcomas.

Treatment was well-tolerated, without the occurrence of toxicity. During therapy, a CT scan indicated a stable disease state after four cycles, and a PR of the lung metastases after 10, 16 and 22 cycles (Fig. 3D). Therapy was continued for 24 cycles (14 months) without relevant side-effects.

In February 2014, 25 months after the diagnosis of metastatic disease and 14 months after the start of the gemcitabine-docetaxel regimen, the patient succumbed to a sudden onset of a cerebral bleeding caused by disease progression in the brain.

\section{Discussion}

EOSs are rare variants of primary osteosarcoma of the bone, and are defined as sarcomas located in the soft tissues and characterized by osteoid production, with no attachment to the skeletal system. The condition was initially described by Wilson in 1941 (23), in the first reported case, and fewer than
300 cases have been reported since then. These cases suggest that aside from the histologically common features, EOS has distinctive demographic, imaging and prognostic features compared with osteosarcoma of bone origin. Furthermore, it is not currently clear whether EOS may be managed in a similar way to osteosarcoma or soft-tissue sarcoma. The rarity of EOSs, in addition to the lack of prospective and randomized studies, has hindered the resolution of this issue.

In all the reported studies of EOS, the importance of a multidisciplinary approach to treatment has been clearly emphasized; such treatment has involved surgery, radiotherapy and chemotherapy, depending on the disease stage. In particular, it has been demonstrated that due to its typical location in the lower extremities, limb-sparing surgical techniques may be applied to patients with EOS, as has been firmly established in cases of bone sarcoma $(1,7,10)$. Furthermore, a wide resection, with R0 status, of surgical margins is one of the most important clinicopathological prognostic factors, in addition to tumor size and tumor-node-metastasis stage $(1-7,10,12)$. Extremely poor experiences have been reported with regard to pre-operative chemotherapy, without a conclusive definition of its modality $(1,6,7,9,10)$. In the adjuvant setting, the largest reported series was that of the Co-operative German-Austrian-Swiss Osteosarcoma Study 
Group, in which 15 patients were treated according to protocols for high-grade central osteosarcoma between 1986 and 2002 (6). In these patients, whose median age was lower than that reported elsewhere, polychemotherapy regimens, including doxorubicin, ifosfamide, cisplatin and methotrexate in certain cases, led to an estimated overall survival rate of $77 \%$ at five years, the highest reported rate of any previous studies. The study concluded that EOS has a favorable prognosis when treated as per conventional osteosarcoma. In another 27 patients with measurable and assessable EOS, treated at the M.D. Anderson Cancer Center between 1960 and 1999, the use of doxorubicin-based chemotherapy, including additional cisplatin in 13 of these patients and ifosfamide in a further 8 , led to a low overall response rate of $19 \%$ (10). In this series, a median survival time of 8 months was documented for metastatic EOS, leading to the conclusion [as previously reported by the same authors (9)] that EOS may be considered to be a relatively chemoresistant form of sarcoma with a poor prognosis, which should be treated distinctly from bone sarcoma.

Following this approach, the patient in the present case was initially treated with a pre-operative soft-tissue sarcoma-oriented chemotherapy regimen $(24,25)$ of epirubicin and ifosfamide, which was found to be ineffective. Subsequently, a different schedule of doxorubicin and cisplatin, which has been well-documented in bone sarcoma (26) was commenced. Although a PR was documented, the patient experienced protracted grade 3 pancytopenia, which necessitated the suspension of the treatment.

As a further relapse of the EOS occurred, the previously experienced severe side-effects were considered during the planning of a new treatment strategy, with the aim of devising a therapy that would be effective against the disease without leading to heavy myelotoxicity. A gemcitabine-docetaxel regimen was therefore selected as salvage therapy. This combination schedule had previously demonstrated synergistic activity against various sarcoma cell lines, including osteosarcoma, in in vitro experiments (14). However, the translation of this evidence into the clinical setting for the treatment of soft-tissue sarcoma was more promising than for bone sarcoma, as phase II trials have demonstrated $(11,15,16,18)$. The role of gemcitabine-docetaxel combination therapy in refractory or recurrent osteosarcoma remains poorly defined due to the relatively small number of patients included in the trials, and due to the retrospective nature of the reports $(13,17,19-22)$.

To the best of our knowledge, the present study was the first clinical experience with this combination regimen for the treatment of EOS. The outcome included an objective PR and a progression-free survival time of 14 months, with a well-tolerated treatment that was easily administered in an outpatient setting.

In conclusion, the lack of novel agents active against these tumors prompted the investigation of a different strategy for the treatment of this rare sarcoma, for which there are currently few therapeutic options. The present case demonstrated that EOS may maintain relative chemosensitivity, indicating the potential to control the advanced disease in the long term and plan sequential chemotherapy regimens.

The establishment of definitive guidelines with regard to the management of EOS has been hampered by the rarity of this sarcoma. In addition, previous studies have been biased by the long retrospective period and poor control over pathological and clinical information. Therefore, it is necessary to strengthen collaborative networks in order to confirm the effectiveness of the gemcitabine-docetaxel combination in large retrospective or prospective studies in EOS patients, for whom established therapeutic strategies are presently lacking.

\section{Acknowledgements}

The authors would like to thank Miss. Caroline Oakley (National Cancer Research Centre, Bari, Italy) and Miss. Silvana Valerio (National Cancer Research Centre, Bari, Italy) for providing assistance in the preparation of this manuscript.

\section{References}

1. McCarter MD, Lewis JJ, Antonescu CR and Brennan MF: Extraskeletal osteosarcoma: Analysis of outcome of a rare neoplasm. Sarcoma 4: 119-123, 2000.

2. Lidang Jensen M, Schumacher B, Myhre Jensen O, Steen Nielsen $\mathrm{O}$ and Keller J: Extraskeletal osteosarcomas: A clinicopathologic study of 25 cases. Am J Surg Pathol 22: 588-594, 1998.

3. Sordillo PP, Hajdu SI, Magill GB and Golbey RB: Extraosseous osteogenic sarcoma. A review of 48 patients. Cancer 51: 727-734, 1983.

4. Bane BL, Evans HL, Ro JY, Carrasco CH, Grignon DJ, Benjamin RS and Ayala AG: Extraskeletal osteosarcoma. A clinicopathologic review of 26 cases. Cancer 65: 2762-2770, 1990.

5. Allan CJ and Soule EH: Osteogenic sarcoma of the somatic soft tissues. Clinicopathologic study of 26 cases and review of literature. Cancer 27: 1121-1133, 1971.

6. Goldstein-Jackson SY, Gosheger G, Delling G, Berdel WE, Exner GU, Jundt G, Machatschek JN, Zoubek A, Jürgens $H$ and Bielack SS; Cooperative Osteosarcoma Study Group COSS: Extraskeletal osteosarcoma has a favourable prognosis when treated like conventional osteosarcoma. J Cancer Res Clin Oncol 131: 520-526, 2005.

7. Torigoe T, Yazawa Y, Takagi T, Terakado A and Kurosawa H: Extraskeletal osteosarcoma in Japan: Multiinstitutional study of 20 patients from the Japanese Musculoskeletal Oncology Group. J Orthop Sci 12: 424-429, 2007.

8. Casali PG: Histology- and non-histology-driven therapy for treatment of soft tissue sarcomas. Ann Oncol 23: x167-x169, 2012.

9. Patel SR and Benjamin RS: Primary extraskeletal osteosarcoma - experience with chemotherapy. J Natl Cancer Inst 87: 1331-1333, 1995.

10. Ahmad SA, Patel SR, Ballo MT, et al: Extraosseous osteosarcoma: Response to treatment and long-term outcome. J Clin Oncol 20: 521-527, 2002.

11. Bay JO, Ray-Coquard I, Fayette J, et al; Groupe Sarcome Français: Docetaxel and gemcitabine combination in 133 advanced soft-tissue sarcomas: a retrospective analysis. Int J Cancer 119: 706-711, 2006.

12. Eisenhauer EA, Therasse P, Bogaerts J, et al: New response evaluation criteria in solid tumours: Revised RECIST guideline (version 1.1). Eur J Cancer 45: 228-247, 2009.

13. Maki RG, Wathen JK, Patel SR, et al: Randomized phase II study of gemcitabine and docetaxel compared with gemcitabine alone in patients with metastatic soft tissue sarcomas: Results of sarcoma alliance for research through collaboration study 002 [corrected]. J Clin Oncol 25: 2755-2763, 2007.

14. Leu KM, Ostruszka LJ, Shewach D, et al: Laboratory and clinical evidence of synergistic cytotoxicity of sequential treatment with gemcitabine followed by docetaxel in the treatment of sarcoma. J Clin Oncol 22: 1706-1712, 2004.

15. Rapkin L, Qayed M, Brill P, Martin M, Clark D, George BA, Olson TA, Wasilewski-Masker K, Alazraki A and Katzenstein HM: Gemcitabine and docetaxel (GEMDOX) for the treatment of relapsed and refractory pediatric sarcomas. Pediatr Blood Cancer 59: 854-858, 2012. 
16. Pautier P, Floquet A, Penel N, et al: Randomized multicenter and stratified phase II study of gemcitabine alone versus gemcitabine and docetaxel in patients with metastatic or relapsed leiomyosarcomas: A Federation Nationale des Centres de Lutte Contre le Cancer (FNCLCC) French Sarcoma Group Study (TAXOGEM study). Oncologist 17: 1213-1220, 2012.

17. Navid F, Willert JR, McCarville MB, Furman W, Watkins A, Roberts W and Daw NC: Combination of gemcitabine and docetaxel in the treatment of children and young adults with refractory bone sarcoma. Cancer 113: 419-425, 2008.

18. Frustaci S, Gherlinzoni F, De Paoli A, et al: Adjuvant chemotherapy for adult soft tissue sarcomas of the extremities and girdles: Results of the Italian randomized cooperative trial. J Clin Oncol 19: 1238-1247, 2001.

19. Hensley ML, Maki R, Venkatraman E, Geller G, Lovegren M, Aghajanian C, Sabbatini P, Tong W, Barakat R and Spriggs DR: Gemcitabine and docetaxel in patients with unresectable leiomyosarcoma: Results of a phase II trial. J Clin Oncol 20 : 2824-2831, 2002.

20. Qi WX, He AN, Tang LN, Shen Z, Lin F and Yao Y: Efficacy and safety of gemcitabine-docetaxel combination therapy for recurrent or refractory high-grade osteosarcoma in China: A retrospective study of 18 patients. Jpn J Clin Oncol 42: 427-431, 2012.
21. He A, Qi W, Huang Y, Sun Y, Shen Z, Zhao H, Yang Y and Yao Y: Comparison of pirarubicin-based versus gemcitabine-docetaxel chemotherapy for relapsed and refractory osteosarcoma: A single institution experience. Int J Clin Oncol 18: 498-505, 2013.

22. Fox E, Patel S, Wathen JK, Schuetze S, Chawla S, Harmon D, Reinke D, Chugh R, Benjamin RS and Helman LJ: Phase II study of sequential gemcitabine followed by docetaxel for recurrent Ewing sarcoma, osteosarcoma, or unresectable or locally recurrent chondrosarcoma: Results of Sarcoma Alliance for Research Through Collaboration Study 003. Oncologist 17: 321,2012

23. Wilson H: Extraskeletal ossifying tumors. Ann Surg 113: 95-112, 1941.

24. Gosiengfiao Y, Reichek J, Woodman J, Ben-Ami T and Walterhouse D: Gemcitabine with or without docetaxel and resection for recurrent osteosarcoma: the experience at Children's Memorial Hospital. J Pediatr Hematol Oncol 34: e63-e65, 2012.

25. Gronchi A, Verderio P, De Paoli A, et al: Quality of surgery and neoadjuvant combined therapy in the ISG-GEIS trial on soft tissue sarcomas of limbs and trunk wall. Ann Oncol 24: 817-823, 2013.

26. Souhami RL, Craft AW, Van der Eijken JW, et al: Randomised trial of two regimens of chemotherapy in operable osteosarcoma: A study of the European Osteosarcoma Intergroup. Lancet 350: 911-917, 1997. 